\title{
Inverse association between triglyceride glucose index and muscle mass in Korean adults: 2008-2011 KNHANES
}

Sung-Ho Ahn ${ }^{1,2}$, Jun-Hyuk Lee ${ }^{1,3^{*+}}$ (D) and Ji-Won Lee ${ }^{1,2^{*+}}$

\begin{abstract}
Background: Since sarcopenia is an important risk factor for falls or cardiovascular disease, early detection and prevention of sarcopenia are being increasingly emphasized. Emerging evidence has indicated relationships between sarcopenia, insulin resistance, and inflammation. The triglyceride glucose (TyG) index, a novel surrogate marker of insulin resistance and systemic inflammation, has not yet been shown to be associated with sarcopenia. This study aimed to examine the relationship between the TyG index and muscle mass in Korean adults.

Methods: This study included 15,741 non-diabetic adults over 19 years old using data from the 2008-2011 Korea National Health and Nutrition Examination Survey. Participants were divided into three groups according to tertiles of the TyG index. A low skeletal muscle mass index (LSMI) was defined by the Foundation for the National Institutes of Health Sarcopenia Project criteria. A weighted multivariate logistic regression model was used to analyze relationships between TyG index tertiles and LSMI.
\end{abstract}

Results: The ORs (95\% Cls) for LSMI in the second and third TyG tertiles, compared to the first tertile, were 1.463 (1.1311.892) and 1.816 (1.394-2.366), respectively, after adjusting for confounding factors. Higher TyG index values were also associated with increased odds of LSMI in adults under 65 years who did not exercise regularly, who consumed less than $30 \mathrm{~g}$ of alcohol per day, who did not currently smoke, and who ate less than $1.5 \mathrm{~g}$ of protein $/ \mathrm{kg} / \mathrm{day}$.

Conclusion: The TyG index was significantly and positively associated with LSMI in Korean adults.

Keywords: Triglyceride and glucose index, Sarcopenia, Low skeletal muscle index, Public health, Insulin resistance, Chronic inflammation, Inflammatory cytokines, Metabolic syndrome

\section{Introduction}

Sarcopenia is defined as an unintentional decline in muscle mass, strength, and performance that arises with aging [1]. However, there are no unified criteria for diagnosing sarcopenia [2-5]. Typically, muscle mass reduces by $3-8 \%$ per decade after the age of 30 , and muscle strength [6, 7], a major part of physical function,

\footnotetext{
*Correspondence: muzzyljh@yuhs.ac; http://orcid.org/0000-0002-1007-1633"; indi5645@yuhs.ac

†Jun-Hyuk Lee and Ji-Won Lee contributed equally to this work.

'Department of Family Medicine, Yonsei University College of Medicine, Seoul, Republic of Korea

Full list of author information is available at the end of the article
}

decreases by $1-2 \%$ per year after 50 years of age $[8,9]$. For this reason, sarcopenia is mainly observed in older adults, although it can also occur in younger adults [10]. The estimated prevalence of sarcopenia has been reported to be at least $4.6 \%$ in all older adults and nearly $25 \%$ in older hospitalized patients [11]. Sarcopenia has become an increasingly important issue in public health as life spans have increased [12, 13]. Indeed, healthcare costs related to sarcopenia were reported to be $\$ 18.5$ billion ( $\$ 10.8$ billion in men, $\$ 7.7$ billion in women) in the United States in 2000, constituting about $1.5 \%$ of total healthcare expenditures for the year [13, 14]. 
Sarcopenia is associated not only to increased risks of falls, fractures, and disabilities $[15,16]$, but also to metabolic disorders, such as insulin resistance, type 2 diabetes mellitus, dyslipidemia, and hypertension [17], as a result of decreases in basal metabolic rate, fat-free mass, and physical activity. Additionally, sarcopenia has been shown to be associated with chronic, low-grade, systemic inflammation [18-21], and several reports have described a relationship between sarcopenia, insulin resistance [22], and inflammation [23].

The triglyceride and glucose index (TyG index), a product of triglyceride levels and fasting plasma glucose concentration, has recently been recognized as a useful clinical surrogate marker of insulin resistance [24, 25] and systemic inflammation [26, 27]. Moreover, the TyG index has been validated for predicting the risk of diabetes and cardiovascular disease syndrome [28-32], as well as for diagnosing metabolic syndrome [33]. However, to date there have been no studies of the relationship between the TyG index and sarcopenia. In this analysis, we investigated the association between TyG index and muscle mass in a representative sample of Korean adults.

\section{Materials and methods}

\section{Study population}

All study data were obtained from the 2008-2011 Korea National Health and Nutrition Examination Survey (KNHANES), a nationwide representative survey conducted by the Korea Centers for Disease Control and
Prevention to assess the health and nutritional status of Koreans. The KNHANES employs a cross-sectional, stratified, multistage, probability sampling design based on age, sex, and geographical area. Sample weights were assigned to subjects to capture a sample representing the general Korean population. Survey items in the KNHANES have been revised partially because of the availability of survey resources, and some questionnaires have been updated and changed over the years [34]. Detailed methods related to the KNHANES have been previously described [34].

A total 37,753 of individuals participated in the 20082011 KNHANES. Of these participants, we excluded 1) those who were under 19 years old $(n=9736), 2)$ those missing serum glucose level data $(n=2772), 3)$ those who did not report history of type 2 diabetes mellitus $(n=937), 4)$ those diagnosed type 2 diabetes mellitus by a doctor, treated with anti-diabetic medications, or treated with insulin $(n=917), 5)$ those who had fasting plasma glucose levels $126 \mathrm{mg} / \mathrm{dL}$ or more $(n=1551), 6)$ those missing body mass index (BMI) data $(n=89), 7)$ those missing serum triglyceride level data $(n=1)$, or 8$)$ those missing whole-body dual-energy X-ray absorptiometry (DXA) data $(n=6369)$. Finally, a total of 15,741 participants (6646 men and 9095 women) were included in this analysis (Fig. 1).

\section{Biochemical measurements}

Blood samples were collected from the antecubital vein from each patient after at least $8 \mathrm{~h}$ of fasting. Serum total

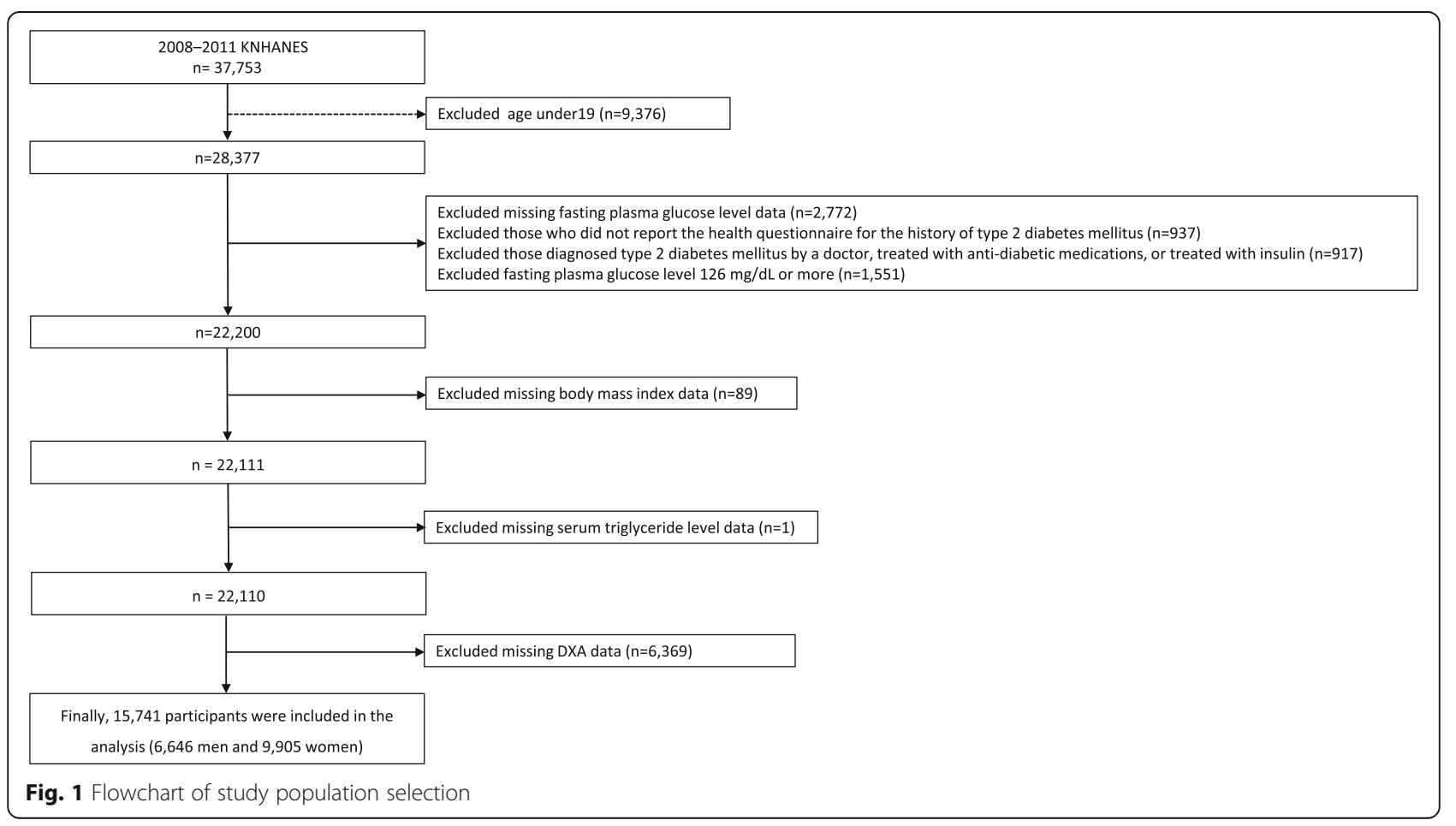


cholesterol, triglycerides, high-density lipoprotein (HDL) cholesterol, and plasma glucose concentrations were measured using a Hitachi 7600 Analyzer. White blood cell (WBC) counts were analyzed using a XE-2100D blood cell counter. The TyG index was calculated as follows: $\log$ [serum triglycerides $(\mathrm{mg} / \mathrm{dL}) \times$ plasma glucose $(\mathrm{mg} / \mathrm{dL}) / 2$ ]. At present, there is no reference value for the prediction of sarcopenia using the TyG index. Therefore, we divided participants into three groups according to tertiles of the TyG index: T1 (6.45-8.19), T2 (8.20-8.71), and T3 (8.72-11.29).

\section{Assessment of muscle mass}

DXA was performed from July 2008 to June 2011 to evaluate body composition (QDR 4500A; Hologic Inc., Bedford, MA, USA). Body composition data were obtained from predefined anatomical areas as follows: head, arms, legs, trunk, pelvic region, and whole body. Participants were analyzed for bone mineral content (g), bone mineral density $\left(\mathrm{g} / \mathrm{cm}^{2}\right)$, fat mass $(\mathrm{g})$, lean body mass $(\mathrm{g})$, and total fat percentage (fat mass/total mass $\times$ 100). Skeletal muscle mass was calculated using the following equation: lean body mass (g) - bone mineral content (g). Appendicular skeletal muscle mass (ASM) was calculated as the summation of the skeletal muscle mass of both upper and lower extremities. We defined the skeletal muscle mass index (SMI) as ASM $(\mathrm{kg})$ divided by body mass index (BMI) $\left(\mathrm{kg} / \mathrm{m}^{2}\right)$. Finally, the low skeletal muscle index (LSMI) was defined according to the Foundation for the National Institutes of Health (FNIH) Sarcopenia Project criteria: SMI values less than 0.789 for men and less than 0.512 for women [5].

\section{Measurement of anthropometric and clinical parameters}

Height $(\mathrm{cm})$ and body weight $(\mathrm{kg})$ were estimated to the nearest $0.1 \mathrm{~cm}$ using a stadiometer without shoes in a standing posture or supine position and to the nearest $0.1 \mathrm{~kg}$ using digital scale in light clothing, respectively. BMI was calculated as body weight divided by height squared $\left(\mathrm{kg} / \mathrm{m}^{2}\right)$. Adults with a BMI greater than or equal to $25 \mathrm{~kg} / \mathrm{m}^{2}$ were considered overweight and those with a BMI less than $18.5 \mathrm{~kg} / \mathrm{m}^{2}$ were considered underweight according to the guidelines of the International Obesity Task Force of the World Health Organization [35]. Adults were categorized into three different categories of smoking status: current smokers, ex-smokers, and never smokers. We defined a current smoker as someone who smoked at the time of the interview and had smoked at least 100 cigarettes over their lifetime. Ex-smokers were defined as those who did not currently smoke, but who had smoked at least 100 cigarettes in his or her lifetime. Heavy alcohol use was defined as consuming an average of $30 \mathrm{~g}$ or more of alcohol per day. We defined regular exercise as $20 \mathrm{~min}$ of vigorous exercise at least 3 days per week or 30 min of moderate exercise/walking at least 5 days per week, and physical activity was assessed using the International Physical Activity Questionnaire (IPAQ). A food frequency questionnaire (FFQ) was used for all adults aged 19 years or older. Daily nutritional intake of total calorie (kcal/day), carbohydrate $(\mathrm{CHO})(\mathrm{g} /$ day), fat $(\mathrm{g} /$ day), and protein $(\mathrm{g} /$ day) were surveyed. Based on the modified NCEP ATPIII criteria [36], this study defined metabolic syndrome according to the following criteria: (1) waist circumference $\geq 90 \mathrm{~cm}$ in men and $\geq 85 \mathrm{~cm}$ in women, per the Korean-specific cut-offs for abdominal obesity of the Korean Society of Obesity [37]; (2) serum triglycerides $\geq 150 \mathrm{mg} / \mathrm{dL}$; (3) either fasting plasma glucose $\geq 100 \mathrm{mg} /$ $\mathrm{dL}$, the use of anti-diabetic medications, or current treatment with insulin therapy; (4) HDL cholesterol $<40 \mathrm{mg} /$ $\mathrm{dL}$ in men or $<50 \mathrm{mg} / \mathrm{dL}$ in women or use of lipidlowering medications; and (5) blood pressure $\geq 130 / 85$ $\mathrm{mmHg}$ or use of anti-hypertensive medications. Detailed information about the KNHANES is available on the KNHANES website (http://knhanes.cdc.go.kr).

\section{Statistical analysis}

All data are presented as a mean or percentage (\%) \pm standard error (SE). For the analysis of clinical characteristics of the study population, a weighted analysis of variance (ANOVA) test was used for continuous variables, followed by post-hoc analysis with Bonferroni correction. For categorical variables, weighted chi-square tests were used to analyze differences among the three groups, followed by post-hoc analysis with Bonferroni correction. After adjusting for confounding variables, a weighted multivariate logistic regression analysis was performed to calculate odds ratios (ORs) with 95\% confidence intervals (CIs) for LSMI according to the TyG index tertiles. We further analyzed subgroups according to age, alcohol drinking status, smoking status, amount of protein intake, and regular exercise through a weighted multivariate logistic regression analysis. All statistical analyses were conducted using SPSS statistical software (version 25.0; SPSS Inc., Chicago, IL, USA). The significance level was set at $p$ less than 0.05 .

\section{Results}

General characteristics of the study population

Table 1 presents the clinical characteristics of 15,741 subjects according to tertiles of the TyG index. The proportions of men and mean age were lowest in $\mathrm{T} 1$ and highest in T3. The mean values of waist circumference, BMI, mean blood pressure, blood leukocyte count, fasting plasma glucose levels, serum total cholesterol levels, and log-transformed triglyceride levels increased, whereas HDL cholesterol levels decreased with each increase in TyG index tertile. The proportions of heavy 
Table 1 Clinical characteristics of three different population

\begin{tabular}{|c|c|c|c|c|c|}
\hline \multirow[t]{2}{*}{ TyG index } & \multicolumn{5}{|c|}{ 2008-2011 KNHANES } \\
\hline & $\mathrm{T} 1$ & $\mathrm{~T} 2$ & T3 & Total & $p$ for trend \\
\hline & $6.45-8.19$ & $8.20-8.71$ & $8.72-11.29$ & & \\
\hline Unweighted number & 5229 & 5235 & 5277 & 15,741 & \\
\hline Male sex,n(\%) & $1520(29.1)$ & $2193(41.9)$ & $2933(55.6)$ & $6641(42.2)$ & $<0.001$ \\
\hline Age, years & $38.3 \pm 0.3^{a}$ & $44.3 \pm 0.3^{b}$ & $47.7 \pm 0.3^{c}$ & $43.4 \pm 0.2$ & $<0.001$ \\
\hline Waist circumference, $\mathrm{cm}$ & $75.2 \pm 0.2^{\mathrm{a}}$ & $80.2 \pm 0.2^{b}$ & $85.5 \pm 0.2^{c}$ & $80.3 \pm 0.1$ & $<0.001$ \\
\hline Body weight, kg & $59.1 \pm 0.2^{a}$ & $63.4 \pm 0.2^{b}$ & $68.2 \pm 0.2^{c}$ & $63.6 \pm 0.1$ & $<0.001$ \\
\hline Mean blood pressure, $\mathrm{mmHg}$ & $85.4 \pm 0.2^{a}$ & $90.3 \pm 0.3^{b}$ & $94.5 \pm 0.3^{c}$ & $90.1 \pm 0.2$ & $<0.001$ \\
\hline Leukocyte count $(* 1000 / \mu \mathrm{L})$ & $5.6 \pm 0.0^{\mathrm{a}}$ & $6.0 \pm 0.0^{b}$ & $6.6 \pm 0.0^{c}$ & $6.1 \pm 0.0$ & $<0.001$ \\
\hline Glucose, mg/dL & $88.0 \pm 0.1^{\mathrm{a}}$ & $92.2 \pm 0.2^{b}$ & $96.9 \pm 0.2^{c}$ & $92.3 \pm 0.1$ & $<0.001$ \\
\hline Log-transformed Triglyceride, $\mathrm{mg} / \mathrm{dL}$ & $4.0 \pm 0.0^{\mathrm{a}}$ & $4.6 \pm 0.0^{b}$ & $5.3 \pm 0.0^{c}$ & $4.7 \pm 0.0$ & $<0.001$ \\
\hline Employment status, \% (SE) & $63.6(0.9)^{\mathrm{a}}$ & $62.9(1.0)^{\mathrm{a}}$ & $68.1(0.9)^{c}$ & $64.8(0.6)$ & $<0.001$ \\
\hline Heavy alcohol use, \% (SE) & $5.2(0.5)^{a}$ & $7.5(0.5)^{b}$ & $13.8(0.7)^{c}$ & $8.8(0.3)$ & $<0.001$ \\
\hline Current smoker, \% (SE) & $18.5(0.8)^{\mathrm{a}}$ & $25.3(0.9)^{b}$ & $38.0(1.0)^{c}$ & $27.0(0.6)$ & $<0.001$ \\
\hline Regular exercise, \% (SE) & $25.5(0.8)^{\mathrm{a}}$ & $25.4(0.8)^{\mathrm{a}}$ & $24.0(0.8)^{a}$ & $25.0(0.5)$ & 0.307 \\
\hline Daily calorie intake, kcal/day & $1982.3 \pm 16.5^{\mathrm{a}}$ & $2010.0 \pm 17.3^{a}$ & $2125.7 \pm 20.1^{c}$ & $2039.3 \pm 11.1$ & $<0.001$ \\
\hline Daily protein intake, $\%$ of total calorie intake & $14.6 \pm 0.1^{\mathrm{a}}$ & $14.4 \pm 0.1^{\mathrm{a}}$ & $14.2 \pm 0.1^{b}$ & $14.4 \pm 0.1$ & 0.001 \\
\hline \multicolumn{6}{|l|}{ SMI } \\
\hline Men & $1.006 \pm 0.004^{\mathrm{a}}$ & $0.963 \pm 0.004^{b}$ & $0.921 \pm 0.003^{c}$ & $0.963 \pm 0.002$ & $<0.001$ \\
\hline Women & $0.666 \pm 0.002^{a}$ & $0.629 \pm 0.002^{b}$ & $0.596 \pm 0.002^{c}$ & $0.631 \pm 0.002$ & $<0.001$ \\
\hline Number of components of metabolic syndrome & & & & & $<0.001$ \\
\hline 0 & $56.3(0.9)^{\mathrm{a}}$ & $30.9(0.9)^{b}$ & $3.4(0.4)^{c}$ & $30.6(0.5)$ & \\
\hline 1 & $33.0(0.8)^{\mathrm{a}}$ & $39.5(0.9)^{b}$ & $15.4(0.7)^{c}$ & $29.3(0.5)$ & \\
\hline 2 & $8.4(0.5)^{\mathrm{a}}$ & $20.0(0.7)^{\mathrm{b}}$ & $29.8(0.9)^{c}$ & $19.2(0.4)$ & \\
\hline 3 & $2.0(0.2)^{a}$ & $8.3(0.4)^{b}$ & $28.9(0.9)^{c}$ & $12.9(0.4)$ & \\
\hline 4 & $0.2(0.1)^{\mathrm{a}}$ & $1.3(0.2)^{\mathrm{b}}$ & $17.8(0.6)^{c}$ & $6.3(0.2)$ & \\
\hline 5 & - & - & $4.8(0.3)$ & $1.6(0.1)$ & \\
\hline Number of chronic diseases, \% (SE) & & & & & $<0.001$ \\
\hline 0 & $95.6(0.4)^{\mathrm{a}}$ & $93.1(0.4)^{b}$ & $92.1(0.5)^{b}$ & $93.5(0.3)$ & \\
\hline 1 & $4.0(0.3)^{a}$ & $6.2(0.4)^{b}$ & $7.2(0.4)^{b}$ & $5.8(0.2)$ & \\
\hline$\geq 2$ & $0.4(0.1)^{a}$ & $0.7(0.1)^{b}$ & $0.7(0.1)^{b}$ & $0.6(0.1)$ & \\
\hline
\end{tabular}

Abbreviations: TyG index triglyceride-glucose index, KNHANES Korean National Health and Nutrition Examination Survey, SE standard error, BMI body mass index, SMI skeletal muscle mass index

$p$ for trend was derived from weighted generalized linear regression analysis for continuous variables, followed by post-hoc analysis with Bonferroni correction and weighted chi-square test for linear-by-linear association for categorical variables, followed by post-hoc analysis with Bonferroni correction.

Superscripts ( $\left.{ }^{\mathrm{a} b \mathrm{c}}\right)$, for a particular variable, indicate significant difference between tertiles $(p<0.0167)$. Variables with the same superscripts are not significantly different $(p>0.0167)$. When only one comparison was significant, one of the cell means has no superscript attached.

alcohol drinkers, current smokers, and number of chronic diseases increased along with TyG index tertiles. The proportion of adults participating in regular exercise was highest in $\mathrm{T} 1$ and lowest in $\mathrm{T} 3$, although these trends were not statistically significant. The average amounts of daily calorie intake, carbohydrate intake, and protein intake significantly increased with increasing TyG index tertiles, while the amount of daily fat intake decreased with increasing TyG index tertiles. The mean values of SMI decreased with increasing TyG index tertiles for both men and women. The prevalence of LSMI increased with increasing TyG index tertiles (Fig. 2). The number of components of metabolic syndrome was higher in T3 than T1. WBC counts increased with an increase in the TyG index tertiles.

\section{Association between TyG index and LSMI}

Table 2 shows the results of multivariate logistic regression analysis, including the ORs (95\% CIs) for LSMI according to the TyG index tertiles. The OR (95\% CIs) for 


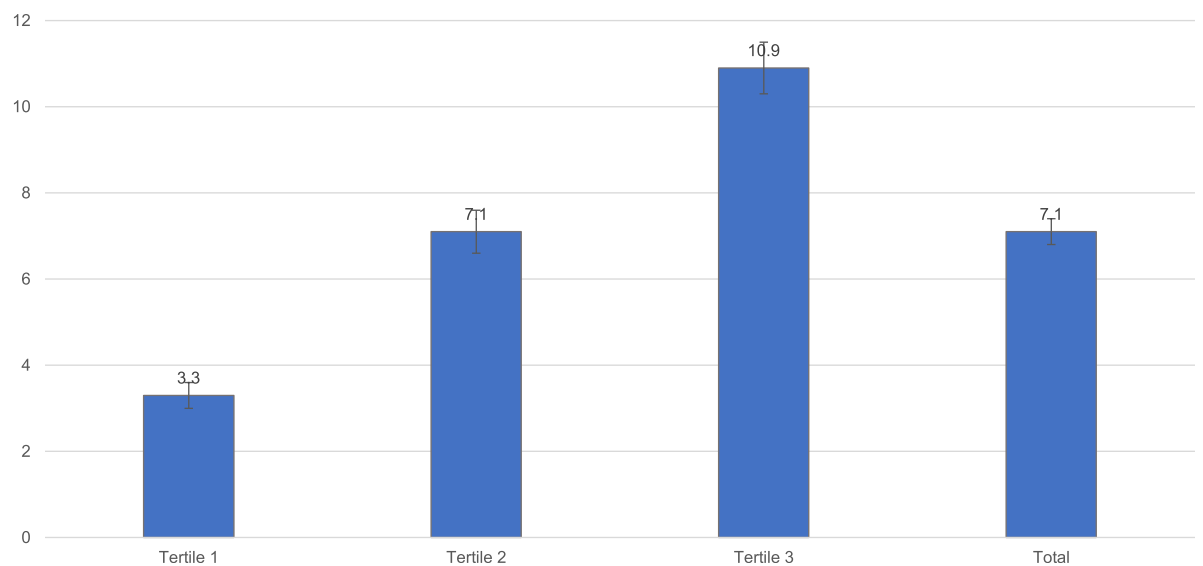

Fig. 2 Prevalence of LSMI according to TyG index tertiles

Table 2 Association between TyG index and LSMI

\begin{tabular}{|c|c|c|c|c|c|c|c|c|c|}
\hline TyG index tertile & Sample size of LSMI, $\mathbf{n}(\%)$ & B & SE & ORs & $95 \% \mathrm{Cls}$ & Wald & $p$ & Overall $p$ & $p$ for trend \\
\hline Unadjusted & & & & & & 79.670 & & $<0.001$ & $<0.001$ \\
\hline $\mathrm{T} 1$ & $228(4.4)$ & Ref. & Ref. & 1 (Ref.) & Ref. & & Ref. & & \\
\hline $\mathrm{T} 2$ & $446(8.5)$ & 0.799 & 79.67 & 2.223 & $1.781-2.776$ & & $<0.001$ & & \\
\hline T3 & $712(13.5)$ & 1.267 & 0.103 & 3.549 & $2.897-4.347$ & & $<0.001$ & & \\
\hline Model 1 & & & & & & 28.277 & & $<0.001$ & $<0.001$ \\
\hline T1 & $228(4.4)$ & Ref. & Ref. & 1 (Ref.) & Ref. & & Ref. & & \\
\hline $\mathrm{T} 2$ & $446(8.5)$ & 0.482 & 0.121 & 1.619 & $1.277-2.053$ & & $<0.001$ & & \\
\hline T3 & $712(13.5)$ & 0.831 & 0.116 & 2.296 & $1.829-2.881$ & & $<0.001$ & & \\
\hline Model 2 & & & & & & 14.880 & & $<0.001$ & $<0.001$ \\
\hline $\mathrm{T} 1$ & $228(4.4)$ & Ref. & Ref. & 1 (Ref.) & Ref. & & Ref. & & \\
\hline $\mathrm{T} 2$ & 446 (8.5) & 0.434 & 0.130 & 1.544 & $1.195-1.994$ & & 0.001 & & \\
\hline $\mathrm{T} 3$ & $712(13.5)$ & 0.711 & 0.133 & 2.037 & $1.569-2.645$ & & $<0.001$ & & \\
\hline Model 3 & & & & & & 10.809 & & $<0.001$ & $<0.001$ \\
\hline $\mathrm{T} 1$ & 228 (4.4) & Ref. & Ref. & 1 (Ref.) & Ref. & & Ref. & & \\
\hline $\mathrm{T} 2$ & $446(8.5)$ & 0.399 & 0.131 & 1.491 & $1.152-1.930$ & & 0.002 & & \\
\hline $\mathrm{T} 3$ & 712 (13.5) & 0.629 & 0.137 & 1.876 & $1.434-2.454$ & & $<0.001$ & & \\
\hline Model 4 & & & & & & 10.013 & & $<0.001$ & $<0.001$ \\
\hline $\mathrm{T} 1$ & $228(4.4)$ & Ref. & Ref. & 1 (Ref.) & Ref. & & Ref. & & \\
\hline $\mathrm{T} 2$ & $446(8.5)$ & 0.380 & 0.131 & 1.463 & $1.131-1.892$ & & 0.004 & & \\
\hline T3 & 712 (13.5) & 0.597 & 0.135 & 1.816 & $1.394-2.366$ & & $<0.001$ & & \\
\hline
\end{tabular}

Abbreviations: TyG index triglyceride-glucose index, LSMI low skeletal muscle mass index, ORs odds ratio, Cls confidence intervals

Model 1: Adjusted for age, sex, body weight, and regular exercise.

Model 2: Adjusted for variables included in Model 1 plus employment status, alcohol use, smoking status, daily protein intake, number of chronic diseases, and serum total cholesterol level.

Model 3: Adjusted for variables included in Model 2 plus plasma glucose level.

Model 4: Adjusted for variables included in Model 3 plus mean blood pressure./

SMI was defined using the following equation: ASM $(\mathrm{kg}) / \mathrm{BMI}(\mathrm{kg} / \mathrm{m} 2)$.

We defined the LSMI according to the cut-off value of SMI based on the Foundation for the National Institutes of Health (FNIH) sarcopenia project criteria: SMI less than 0.789 for men and SMI less than 0.512 for women.

The odds ratio and 95\% confidence interval were calculated using the weighted multi-variate logistic regression analysis to evaluate the relationship between TyG index and LSMI. 
LSMI in T3 of the TyG index versus T1 was 3.549 (2.897-4.347). This relationship remained significant after adjusting for age, sex, body weight, regular exercise, employment status, heavy alcohol use, smoking status, daily protein intake, number of chronic diseases, total cholesterol level, plasma glucose level, and mean blood pressure (T3 vs. T1, ORs $=1.816$, 95\% CIs: $1.394-2.366, p<0.001)$.

Table 3 presents the results of subgroup analysis showing the relationship between TyG index and LSMI. The fully adjusted ORs (95\% CIs) for LSMI in T3 compared to T1 were significant in both sex, adults under 65 years of age, both regular and irregular exercising groups, adults with normal body weight, drinking alcohol less than $30 \mathrm{~g}$ per day group, both current smokers and non-current smokers, both adults who consumed $1.5 \mathrm{~g}$ per $\mathrm{kg}$ of protein or more per day and adults who consumed less than $1.5 \mathrm{~g}$ per $\mathrm{kg}$ of protein per day. There were no significant differences between groups among adults over 65 years of age, underweight adults, overweight adults, and heavy alcohol drinkers.

Each $P$ was calculated by multivariate logistic regression analysis after adjusting for all confounders (age, sex, body weight, regular exercise, employment status, alcohol use, smoking status, daily protein intake, number of chronic diseases, serum total cholesterol level, and plasma glucose level, and mean blood pressure), except for the variable used in each subgroup analysis.

\section{Discussion}

The prevention of frailty is an important strategy through which to address related comorbidities, such as falls, cardiovascular events, cognitive impairment, and mortality [38, 39]. The cycle of frailty consists of four main components: reduced resting metabolic rate, decreased total energy expenditure, chronic undernutrition, and sarcopenia [40]. Of these, sarcopenia is of particular importance to enabling activities of daily living, preventing falls, and reducing various metabolic diseases $[39,40]$. Therefore, methods for the early detection and prevention of sarcopenia would be of clinical and societal value.

In this study, adults of both sexes who had higher TyG index values were more likely to have LSMI, even after adjusting for age and other confounding factors. These findings were based on data from a representative,

Table 3 Subgroup analysis for relationships between TyG index tertiles and LSMI using multivariate logistic regression analysis

\begin{tabular}{|c|c|c|c|c|c|c|c|}
\hline & T1 & $\begin{array}{l}\text { T2 } \\
\text { ORs (95\% Cls) }\end{array}$ & $p$ & $\begin{array}{l}\text { T3 } \\
\text { ORs (95\% Cls) }\end{array}$ & $p$ & overall $p$ & $p$ for trend \\
\hline \multicolumn{8}{|l|}{ Sex } \\
\hline Men & 1 (Ref.) & 1. $485(0.969-2.275)$ & 0.069 & $2.138(1.377-3.319)$ & 0.001 & 0.001 & $<0.001$ \\
\hline Women & 1 (Ref.) & $1.415(1.068-1.874)$ & 0.016 & $1.620(1.189-2.207)$ & 0.002 & 0.010 & 0.003 \\
\hline \multicolumn{8}{|l|}{ Age groups } \\
\hline$<65$ & 1 (Ref.) & $1.864(1.363-2.549)$ & $<0.001$ & 2.691 (1.947-3.719) & $<0.001$ & $<0.001$ & $<0.001$ \\
\hline$\geq 65$ & 1 (Ref.) & $1.177(0.823-1.683)$ & 0.371 & $1.333(0.940-1.889)$ & 0.107 & 0.248 & 0.095 \\
\hline \multicolumn{8}{|l|}{ Regular exercise } \\
\hline Yes & 1 (Ref.) & $1.628(0.925-2.866)$ & 0.091 & $1.862(1.074-3.229)$ & 0.027 & 0.087 & 0.027 \\
\hline No & 1 (Ref.) & $1.400(1.068-1.833)$ & 0.015 & $1.795(1.352-2.384)$ & $<0.001$ & $<0.001$ & $<0.001$ \\
\hline \multicolumn{8}{|l|}{ Weight status } \\
\hline Underweight & 1 (Ref.) & $1.201(0.205-7.027)$ & 0.839 & $0.085(0.005-1.432)$ & 0.087 & 0.228 & 0.132 \\
\hline Normal & 1 (Ref.) & $1.412(1.018-1.959)$ & 0.039 & $1.568(1.085-2.266)$ & 0.017 & 0.050 & 0.021 \\
\hline Overweight & 1 (Ref.) & $0.990(0.685-1.430)$ & 0.957 & $1.060(0.747-1.505)$ & 0.743 & 0.835 & 0.621 \\
\hline \multicolumn{8}{|l|}{ Heavy alcoholics } \\
\hline$<30$ g/day & 1 (Ref.) & $1.535(1.206-1.954)$ & 0.001 & $1.897(1.470-2.448)$ & $<0.001$ & $<0.001$ & $<0.001$ \\
\hline$\geq 30$ g/day & 1 (Ref.) & $0.334(0.088-1.266)$ & 0.107 & $0.668(0.180-2.483)$ & 0.547 & 0.133 & 0.997 \\
\hline \multicolumn{8}{|l|}{ Current smoker } \\
\hline Yes & 1 (Ref.) & $1.167(0.624-2.183)$ & 0.629 & $2.097(1.096-4.012)$ & 0.025 & 0.009 & 0.008 \\
\hline No & 1 (Ref.) & 1.536 (1.190-1.983) & 0.001 & $1.775(1.363-2.313)$ & $<0.001$ & $<0.001$ & $<0.001$ \\
\hline \multicolumn{8}{|l|}{ Protein intake } \\
\hline$>1.5$ g/kg/day & 1 (Ref.) & $1.133(0.582-2.202)$ & 0.713 & $2.160(1.132-4.121)$ & 0.020 & 0.008 & 0.010 \\
\hline$\leq 1.5 \mathrm{~g} / \mathrm{kg} / \mathrm{day}$ & 1 (Ref.) & $1.515(1.151-1.995)$ & 0.003 & $1.816(1.361-2.423)$ & $<0.001$ & $<0.001$ & $<0.001$ \\
\hline
\end{tabular}


nationwide, cross-sectional survey. The exact mechanism by which TyG index is positively associated with LSMI is not known, although we hypothesize that insulin resistance and chronic inflammation may be the major links between elevated TyG index and an increased risk of sarcopenia. The pathogenesis of sarcopenia has been suggested to be closely related to chronic inflammation [20], which consequently increases insulin resistance [41], and may be reflected as an increased TyG index. The TyG index is thought to represent insulin resistance because it is calculated on the basis of two metabolic parameters: serum triglycerides and fasting glucose. Although we could not directly investigate the association between TyG index and insulin resistance index due to a lack of insulin data in the 2008-2011 KNHANES, we did find that the TyG index was related to the severity of metabolic syndrome, which is closely associated with insulin resistance [24, 42]. In addition, we found that a higher TyG index was associated with higher blood leukocyte count, a marker of chronic inflammation [43].

Sarcopenia is accompanied by muscle fat accumulation and an increase in pro-inflammatory cytokines, such as interleukin-6 (IL-6) and tissue necrosis factor alpha (TNF$\alpha)$ within myocytes [44, 45], which contribute to subsequent decreases in muscle mass and strength [46, 47]. IL6 downregulates glucose transporter 4 expression and insulin receptor substrate-1 (IRS-1), resulting in reduced transport of glucose into cells (including myocytes) and aggravation of insulin resistance [48]. TNF- $\alpha$ initiates a wide range of downstream signaling cascades, such as the activation of nuclear factor kappa B (NF- $\mathrm{kB}$ ) and c-Jun Nterminal kinase (JNK) $[49,50]$. In turn, NF- $k B$ and JNK lead to the impairment of IRS-1 and aggravation of insulin resistance [49]. Moreover, upregulated NF- $\kappa B$ caused by pro-inflammatory cascades causes ubiquitination of muscle proteins and dissociation of actin and myosin filaments, which consequently leads to further loss of skeletal muscle [51, 52].

In subgroup analysis, the TyG index was independently related to LSMI regardless of the presence of several risk factors for sarcopenia, such as sex, smoking status, and protein intake status [53-55]. When we divided study subjects according to age, adults under 65 years had higher odds of LSMI with increasing higher TyG index tertile; however, there was no relationship between TyG index and LSMI in adults over 65 years of age. Although we could not explain the exact cause, the different percentage of participants with at least one chronic disease could account for the difference between younger and old adults (4.2\% in younger adults group vs. $21.3 \%$ in older adult group). Regular exercise has positive effects on muscle mass and decrease insulin resistance and chronic inflammation [56, 57]. In our study, the regular exercise group showed a weakened relationship between TyG and LSMI (overall $P=0.087$ ), compared to the non-regular exercise group. However, the ORs for LSMI showed consistent linearity with increases in TyG index tertile in both groups $(P$ for trend $=0.027$, < 0.001). Because the IPAQ does not provide information on the timing of exercise and or type/duration of physical activity, further research is needed with more detailed information on physical activity.

The relationship between the TyG index and LSMI was only significant in the normal alcohol consumption group $(<30 \mathrm{~g} /$ day $)$. Prior evidence has suggested that heavy alcohol drinking may accelerate sarcopenia [58], and it may interfere with the relationship between TyG index and LSMI in the excessive consumption group ( $\geq 30 \mathrm{~g} /$ day). When we divided the study group according to BMI, only the normal weight group showed a significant relationship between TyG index tertile and LSMI. Obese people are at risk for being exposed to oxidative stress and chronic inflammation [59], and this chronic inflammatory status could contribute to TyG index values in various ways, such as glucose uptake and adipokine secretion [60,61]. The number of individuals in the underweight group was small, and small sample size could act as confounding factor in the statistical analysis. Further research with a larger number of study participants would be helpful to elucidating the relationship between TyG index and LSMI in underweight individuals.

Unlike aging, alcohol drinking, exercise, underweight, and obesity are modifiable risk factors. Therefore, reducing alcohol consumption, regular exercising, and maintaining normal weight could be priority strategies to preventing LSMI.

\section{Strengths and limitations}

This is the first study to confirm a relationship between the TyG index and muscle mass through the use of DXA and a representative, nationwide dataset from Korean adults. However, this study had several limitations. First, data on muscle mass information was only collected; this study could not obtain muscle strength or performance data, precluding a direct diagnosis of sarcopenia among the adults. Second, the study could not compare TyG index values with insulin resistance index values, such as homeostatic model assessment for insulin resistance. Finally, due to the cross-sectional study design, we could not assess causality between TyG index and LSMI.

\section{Conclusion}

In conclusion, this study found that the TyG index is independently and negatively associated with LSMI in Korean adults over 19 years old. Because the TyG index can be easily measured in clinical settings through blood 
sampling, this may serve as a helpful method for the early detection of sarcopenia and related comorbidities, thus enabling timely initiation of treatment and reduced social, healthcare expenditures for treating sarcopenia. Longitudinal cohort studies and exploratory studies are needed to investigate the relationship between the TyG index and sarcopenia.

\section{Supplementary Information}

The online version contains supplementary material available at https://doi. org/10.1186/s12944-020-01414-4.

Additional file 1. Table S1. Clinical characteristics of the study population without dual energy X-ray absorptiometry data. Table S2. Clinical characteristics of the study population with or without dual energy $\mathrm{X}$-ray absorptiometry data.

\section{Abbreviations}

TyG: Triglyceride-glucose; LSMI: Low skeletal muscle mass index; KNHA NES: Korea National Health and Nutrition Examination Survey; DXA: Dualenergy X-ray absorptiometry; HDL: High-density lipoprotein; WBC: White blood cell; ASM: Appendicular skeletal muscle mass; SMI: Skeletal muscle mass index; BMI: Body mass index; FNIH: Foundation for the National Institutes of Health; IPAQ: International Physical Activity Questionnaire; FFQ: Food frequency questionnaire; CHO: Carbohydrate; SE: Standard error; ORs: Odds ratios; Cis: Confidence intervals; IL-6: Interleukin-6; TNF- a: Tissue necrosis factor alpha; IRS-1: Insulin receptor substrate-1; NF-kB: Nuclear factor kappa B; JNK: c-Jun N-terminal kinase

\section{Acknowledgments}

This work was supported by the 2020 faculty research grant from Yonsei University College of Medicine (6-2020-0143) and the Technology Innovation Program (20002781, A Platform for Prediction and Management of Health Risk Based on Personal Big Data and Lifelogging) funded by the Ministry of Trade, Industry \& Energy (MOTIE, Korea).

\section{Conflict of interest}

The authors have no conflicts of interest related to this report.

\section{Financial disclosure}

The authors have no financial disclosures related to this report.

\section{Authors' contributions}

SHA and JHL made substantial contributions to conception, design of the study. JWL contributed to the study design. SHA and JWL contributed to the acquisition and interpretation of the data. SHA and JHL performed the statistical analysis. SHA prepared the first version of the manuscript. JHL and JWL revised the manuscript critically for essential intellectual content. All authors read and approved the final version to be published.

\section{Funding}

This research did not receive any specific funding from agencies in the public, commercial, or not-for-profit sectors.

\section{Availability of data and materials}

The dataset used in this study (KNHANES) can be obtained from the Korea Centers for Disease Control and Prevention (http://www.cdc.go.kr/CDC/eng/ main.jsp) after submission and evaluation of an appropriate research proposal.

\section{Ethics approval and consent to participate}

Informed consent was obtained from all eligible participants. Institutional Review Board approval was not required because the survey did not deal with any sensitive information, only publicly available information.

\section{Consent for publication}

Sung-Ho Ahn, Jun-Hyuk Lee, and Ji-Won Lee read and approved to publication.

\section{Competing interests}

Sung-Ho Ahn, Jun-Hyuk Lee, and Ji-Won Lee read and approved to publication.

\section{Author details}

'Department of Family Medicine, Yonsei University College of Medicine, Seoul, Republic of Korea. ${ }^{2}$ Department of Family Medicine, Yonsei University College of Medicine, Gangnam Severance Hospital, 211 Eonju-ro, Gangnam-gu, Seoul 06273, Republic of Korea. ${ }^{3}$ Department of Family Medicine, Yonsei University College of Medicine, Yongin Severance Hospital, 363, Dongbaekjukjeon-daero, Giheung-gu, Yongin-si, Gyeonggi-do 16995, Republic of Korea.

Received: 16 September 2020 Accepted: 2 November 2020

Published online: 22 November 2020

\section{References}

1. Morley JE, Baumgartner RN, Roubenoff R, Mayer J, Nair KS. Sarcopenia. J Lab Clin Med. 2001;137(4):231-43.

2. Cruz-Jentoft AJ, Baeyens JP, Bauer JM, Boirie Y, Cederholm T, Landi F, et al. Sarcopenia: European consensus on definition and diagnosis: Report of the European Working Group on Sarcopenia in Older People. Age Ageing. 2010; 39(4):412-23.

3. Fielding RA, Vellas B, Evans WJ, Bhasin S, Morley JE, Newman AB, et al. Sarcopenia: an undiagnosed condition in older adults. Current consensus definition: prevalence, etiology, and consequences. International working group on sarcopenia. J Am Med Dir Assoc. 2011;12(4):249-56.

4. Chen LK, Liu LK, Woo J, Assantachai P, Auyeung TW, Bahyah KS, et al. Sarcopenia in Asia: consensus report of the Asian Working Group for Sarcopenia. J Am Med Dir Assoc. 2014;15(2):95-101.

5. Studenski SA, Peters KW, Alley DE, Cawthon PM, McLean RR, Harris TB, et al, The FNIH sarcopenia project: rationale, study description, conference recommendations, and final estimates. J Gerontol A Biol Sci Med Sci. 2014; 69(5):547-58.

6. Holloszy JO. The biology of aging. Mayo Clin Proc. 2000;75 Suppl:S3-8; discussion S-9.

7. Melton L, 3rd, Khosla S, Crowson CS, O'Connor MK, O'Fallon WM, Riggs BL. Epidemiology of sarcopenia. J Am Geriatr Soc. 2000;48(6):625-30.

8. Harris T. Muscle mass and strength: relation to function in population studies. J Nutr. 1997;127(5 Suppl):1004S-6S.

9. Metter EJ, Conwit R, Tobin J, Fozard JL. Age-associated loss of power and strength in the upper extremities in women and men. J Gerontol A Biol Sci Med Sci. 1997;52(5):B267-76.

10. Shafiee G, Keshtkar A, Soltani A, Ahadi Z, Larijani B, Heshmat R. Prevalence of sarcopenia in the world: a systematic review and meta- analysis of general population studies. J Diabetes Metab Disord. 2017;16:21.

11. Patel HP, Syddall HE, Jameson $\mathrm{K}$, Robinson S, Denison H, Roberts $\mathrm{HC}$, et al. Prevalence of sarcopenia in community-dwelling older people in the UK using the European Working Group on Sarcopenia in Older People (EWGSOP) definition: findings from the Hertfordshire Cohort Study (HCS). Age Ageing. 2013;42(3):378-84.

12. Bruyère $O$, Beaudart $C$, Locquet $M$, Buckinx F, Petermans J, Reginster JY. Sarcopenia as a public health problem. European Geriatric Medicine. 2016 7(3):272-5.

13. Beaudart C, Rizzoli R, Bruyere O, Reginster JY, Biver E. Sarcopenia: burden and challenges for public health. Arch Public Health. 2014;72(1):45.

14. Janssen I, Shepard DS, Katzmarzyk PT, Roubenoff R. The healthcare costs of sarcopenia in the United States. J Am Geriatr Soc. 2004;52(1):80-5.

15. Yeung SSY, Reijnierse EM, Pham VK, Trappenburg MC, Lim WK, Meskers CGM, et al. Sarcopenia and its association with falls and fractures in older adults: A systematic review and meta-analysis. J Cachexia Sarcopenia Muscle. 2019;10(3):485-500.

16. Benjumea AM, Curcio CL, Duque G, Gomez F. Dynapenia and Sarcopenia as a Risk Factor for Disability in a Falls and Fractures Clinic in Older Persons. Open Access Maced J Med Sci. 2018;6(2):344-9.

17. Karakelides H, Nair KS. Sarcopenia of aging and its metabolic impact. Curr Top Dev Biol. 2005;68:123-48. 
18. Dalle S, Rossmeislova L, Koppo K. The Role of Inflammation in Age-Related Sarcopenia. Front Physiol. 2017;8:1045.

19. Meng SJ, Yu LJ. Oxidative stress, molecular inflammation and sarcopenia. Int J Mol Sci. 2010;11(4):1509-26

20. Beyer I, Mets T, Bautmans I. Chronic low-grade inflammation and agerelated sarcopenia. Curr Opin Clin Nutr Metab Care. 2012;15(1):12-22.

21. Jensen GL. Inflammation: roles in aging and sarcopenia. JPEN J Parenter Enteral Nutr. 2008;32(6):656-9.

22. Du Y, Oh C, No J. Associations between Sarcopenia and Metabolic Risk Factors: A Systematic Review and Meta-Analysis. J Obes Metab Syndr. 2018; 27(3):175-85.

23. Schaap LA, Pluijm SM, Deeg DJ, Visser M. Inflammatory markers and loss of muscle mass (sarcopenia) and strength. Am J Med. 2006;119(6):526 e9-17.

24. Simental-Mendia LE, Rodriguez-Moran M, Guerrero-Romero F. The product of fasting glucose and triglycerides as surrogate for identifying insulin resistance in apparently healthy subjects. Metab Syndr Relat Disord. 2008; 6(4):299-304.

25. Guerrero-Romero F, Simental-Mendia LE, Gonzalez-Ortiz M, MartinezAbundis E, Ramos-Zavala MG, Hernandez-Gonzalez SO, et al. The product of triglycerides and glucose, a simple measure of insulin sensitivity. Comparison with the euglycemic-hyperinsulinemic clamp. J Clin Endocrinol Metab. 2010;95(7):3347-51.

26. Park K, Ahn CW, Lee SB, Kang S, Nam JS, Lee BK, et al. Elevated TyG index predicts progression of coronary artery calcification. Diabetes Care. 2019; 42(8):1569-73.

27. Mazidi M, Kengne AP, Katsiki N, Mikhailidis DP, Banach M. Lipid accumulation product and triglycerides/glucose index are useful predictors of insulin resistance. J Diabetes Complications. 2018;32(3):266-70.

28. Sanchez-Inigo L, Navarro-Gonzalez D, Fernandez-Montero A, PastranaDelgado J, Martinez JA. The TyG index may predict the development of cardiovascular events. Eur J Clin Invest. 2016;46(2):189-97.

29. Lee SH, Kwon HS, Park YM, Ha HS, Jeong SH, Yang HK, et al. Predicting the development of diabetes using the product of triglycerides and glucose: the Chungju Metabolic Disease Cohort (CMC) study. PLoS One. 2014;9(2): e90430.

30. Navarro-Gonzalez D, Sanchez-Inigo L, Pastrana-Delgado J, FernandezMontero A, Martinez JA. Triglyceride-glucose index (TyG index) in comparison with fasting plasma glucose improved diabetes prediction in patients with normal fasting glucose: The Vascular-Metabolic CUN cohort. Prev Med. 2016:86:99-105.

31. Li M, Zhan A, Huang $X$, Hu L, Zhou W, Wang T, et al. Positive association between triglyceride glucose index and arterial stiffness in hypertensive patients: the China H-type Hypertension Registry Study. Cardiovasc Diabetol. 2020;19(1):139.

32. da Silva A, Caldas APS, Rocha D, Bressan J. Triglyceride-glucose index predicts independently type 2 diabetes mellitus risk: A systematic review and meta-analysis of cohort studies. Prim Care Diabetes. 2020.

33. Shin K-AJBSL. Triglyceride and glucose (TyG) index is a clinical surrogate marker for the diagnosis of metabolic syndrome. Biomed Sci Lett. 2017; 23(4):348-54.

34. Kweon S, Kim Y, Jang MJ, Kim Y, Kim K, Choi S, et al. Data resource profile: the Korea National Health and Nutrition Examination Survey (KNHANES). Int J Epidemiol. 2014;43(1):69-77.

35. World Health Organization. Regional Office for the Western P. The AsiaPacific perspective : redefining obesity and its treatment: Sydney: Health Communications Australia; 2000.

36. Grundy SM, Cleeman II, Daniels SR, Donato KA, Eckel RH, Franklin BA, et al. Diagnosis and management of the metabolic syndrome: an American Heart Association/National Heart, Lung, and Blood Institute Scientific Statement. Circulation. 2005;112(17):2735-52

37. Lee SY, Park HS, Kim DJ, Han JH, Kim SM, Cho GJ, et al. Appropriate waist circumference cutoff points for central obesity in Korean adults. Diabetes Res Clin Pract. 2007;75(1):72-80.

38. Rockwood K, Stadnyk K, MacKnight C, McDowell I, Hebert R, Hogan DB. A brief clinical instrument to classify frailty in elderly people. Lancet. 1999; 353(9148):205-6.

39. Speechley $M$, Tinetti $M$. Falls and injuries in frail and vigorous community elderly persons. J Am Geriatr Soc. 1991;39(1):46-52

40. Fried LP, Tangen CM, Walston J, Newman AB, Hirsch C, Gottdiener J, et al. Frailty in older adults: evidence for a phenotype. J Gerontol A Biol Sci Med Sci. 2001;56(3):M146-56.
41. Rehman K, Akash MS. Mechanisms of inflammatory responses and development of insulin resistance: how are they interlinked? J Biomed Sci. 2016;23(1):87.

42. Unger G, Benozzi SF, Perruzza F, Pennacchiotti GL. Triglycerides and glucose index: a useful indicator of insulin resistance. Endocrinol Nutr. 2014;61(10): 533-40.

43. Chmielewski PP, Strzelec B. Elevated leukocyte count as a harbinger of systemic inflammation, disease progression, and poor prognosis: a review. Folia Morphol (Warsz). 2018;77(2):171-8.

44. Krebs M, Roden M. Molecular mechanisms of lipid-induced insulin resistance in muscle, liver and vasculature. Diabetes Obes Metab. 2005;7(6): 621-32.

45. Guo ZK. Intramyocellular lipid kinetics and insulin resistance. Lipids Health Dis. 2007:6:18

46. Aleman $\mathrm{H}$, Esparza J, Ramirez FA, Astiazaran $H$, Payette $H$. Longitudinal evidence on the association between interleukin-6 and C-reactive protein with the loss of total appendicular skeletal muscle in free-living older men and women. Age Ageing. 2011;40(4):469-75.

47. Bautmans I, Onyema O, Van Puyvelde K, Pleck S, Mets T. Grip work estimation during sustained maximal contraction: validity and relationship with dependency and inflammation in elderly persons. J Nutr Health Aging. 2011;15(8):731-6.

48. Chen L, Chen R, Wang $H$, Liang F. Mechanisms linking inflammation to insulin resistance. Int J Endocrinol. 2015;2015:508409.

49. Aguirre V, Uchida T, Yenush L, Davis R, White MF. The c-Jun NH(2)-terminal kinase promotes insulin resistance during association with insulin receptor substrate-1 and phosphorylation of Ser(307). J Biol Chem. 2000;275(12): 9047-54.

50. Chen G, Goeddel DV. TNF-R1 signaling: a beautiful pathway. Science. 2002; 296(5573):1634-5.

51. Powers SK, Kavazis AN, McClung JM. Oxidative stress and disuse muscle atrophy. J Appl Physiol (1985). 2007;102(6):2389-97.

52. Buford TW, Cooke MB, Manini TM, Leeuwenburgh C, Willoughby DS. Effects of age and sedentary lifestyle on skeletal muscle NF-kappaB signaling in men. J Gerontol A Biol Sci Med Sci. 2010;65(5):532-7.

53. Paddon-Jones D, Rasmussen BB. Dietary protein recommendations and the prevention of sarcopenia. Curr Opin Clin Nutr Metab Care. 2009:12(1):86-90.

54. Rom O, Kaisari S, Aizenbud D, Reznick AZ. Sarcopenia and smoking: a possible cellular model of cigarette smoke effects on muscle protein breakdown. Ann N Y Acad Sci. 2012;1259:47-53.

55. Messier V, Rabasa-Lhoret R, Barbat-Artigas S, Elisha B, Karelis AD, AubertinLeheudre M. Menopause and sarcopenia: A potential role for sex hormones. Maturitas. 2011;68(4):331-6.

56. Phu S, Boersma D, Duque G. Exercise and Sarcopenia. J Clin Densitom. 2015 18(4):488-92.

57. Song DS, Chang UI, Choi S, Jung YD, Han K, Ko SH, et al. Heavy Alcohol Consumption with Alcoholic Liver Disease Accelerates Sarcopenia in Elderly Korean Males: The Korean National Health and Nutrition Examination Survey 2008-2010. PLoS One. 2016;11(9):e0163222.

58. Furukawa $S$, Fujita $T$, Shimabukuro $M$, Iwaki $M$, Yamada $Y$, Nakajima $Y$, et al. Increased oxidative stress in obesity and its impact on metabolic syndrome. J Clin Invest. 2004;114(12):1752-61.

59. Maddux BA, See W, Lawrence JC, Jr., Goldfine AL, Goldfine ID, Evans JL. Protection against oxidative stress-induced insulin resistance in rat $\mathrm{L} 6$ muscle cells by mircomolar concentrations of alpha-lipoic acid. Diabetes. 2001;50(2):404-10.

60. Rudich A, Tirosh A, Potashnik R, Hemi R, Kanety H, Bashan N. Prolonged oxidative stress impairs insulin-induced GLUT4 translocation in 3T3-L1 adipocytes. Diabetes. 1998;47(10):1562-9.

61. Montero-Fernandez N, Serra-Rexach JA. Role of exercise on sarcopenia in the elderly. Eur J Phys Rehabil Med. 2013:49(1):131-43.

\section{Publisher's Note}

Springer Nature remains neutral with regard to jurisdictional claims in published maps and institutional affiliations. 